\title{
Maternal factors are important predictors for surgical site infection following cesarean section in Northwest Ethiopian
}

\author{
Oumer Ali $^{\text {a }}$, Dawit Kassahun ${ }^{a}$, Bayew Kelkay Rade ${ }^{\text {b,* }}$, Asmamaw Atnafuc \\ ${ }^{a}$ Department of Gynecology and Obstetrics, School of Medicine, College of Medicine and Health Sciences, University of Gondar, Gondar, Ethiopia \\ ${ }^{\mathrm{b}}$ Department of General Midwifery, School of Midwifery, College of Medicine and Health Sciences, University of Gondar, Gondar, Ethiopia \\ ${ }^{\mathrm{c}}$ Department of Health System and Policy, Institute of Public Health, College of Medicine and Health Sciences, University of Gondar, Gondar, Ethiopia
}

\section{A R T I C L E I N F O}

\section{Keywords:}

Cesarean delivery

Maternal factors

Surgical site infection

\begin{abstract}
A B S T R A C T
Background: Surgical site infection (SSI) is a common surgical complication following cesarean section(C/S) that increases maternal morbidity, duration of hospital stay and the cost of treatment. It is more prevalent in SubSaharan Countries, including Ethiopia. As the best of authors knowledge, limited representative data in the study setting. Therefore, this study aimed to figure out the magnitude and factors associated with surgical site infection.

Methods: Client record review was employed from 818 mothers who underwent cesarean section in University of Gondar comprehensive specialized hospital from January 1, 2018 to January 1, 2019. Charts were selected using systematic random sampling technique. Data were entered into EPI info version 7.0 and then exported to SPSS version 20 for analysis. Descriptive statistics, bivariate, and multivariable logistic regression were applied to summarize the data. A p-value $<0.05$ was a cut-off point to declare statistical significance.

Results: This study revealed that the prevalence of SSI was $12.2 \%$ with $95 \%$ CI (9.96-14.44). Chorioamnionitis [AOR $=6.46,95 \%$ CI (1.82-22.71)], diabetes mellitus [AOR =6.02, 95\% CI (1.69-21.36)], rupture of membrane more than $12 \mathrm{~h}$ before cesarean section [AOR $=2.94,95 \% \mathrm{CI}(1.52-5.67)]$, meconium-stained amniotic fluid $[\mathrm{AOR}=2.43,95 \% \mathrm{CI}(1.23-4.81)]$ and anemia $[\mathrm{AOR}=3.44,95 \% \mathrm{CI}(1.56-7.56)]$ were significantly associated factors with SSI following cesarean delivery.

Conclusion: The magnitude of SSI was high, and maternal related (medical and obstetrical) factors were found significant association with it. Improvement of early identification of risk factors, and prompt treatment/control of obstetrical and medical complications are the recommendations.
\end{abstract}

\section{Introduction}

According to the Centers for Disease Control and Prevention (CDC); surgical site infection (SSI) is defined as infection which occurs within 30 days after surgical procedure involving skin, subcutaneous tissue, soft tissue, or any other part of the anatomy as being either incisional or organ space. ${ }^{1}$ Incisional SSIs are divided into superficial incision those involving only skin and subcutaneous tissue, deep incisional SSI which involved the deeper soft tissue of the incision and organ/space involves any part of the anatomy other than incisional body wall layers. ${ }^{2}$ SSI is common surgical complication among patients delivered with cesarean section(C/S) which increase maternal morbidity, stay of hospital and the cost of treatment. ${ }^{3}$ According to World Health Organization (WHO) global health report (2014), direct (obstetric) infections are the third most common cause of maternal mortality, representing about $10.7 \%$ of maternal deaths, with the largest toll estimated in low-income and middle-income countries (LMICs) at 10.7\% compared with high-income countries (HICs) at $4.7 \%$.

Cesarean delivery is effective in saving maternal and infant life, but only done with obstetric indications (to mother or baby need) and the ideal rate for this procedure to be between $10 \%$ and $15 \% .{ }^{4}$ In 2015, an estimated 29.7 million (21.1\%) births globally were by CS. ${ }^{5}$ According to World Congress of Gynecology and Obstetrics; 6.2 million unnecessary cesareans done each year. ${ }^{6}$ A review study conducted in Sub-Saharan African countries showed that the overall cesarean section (CS) rate was $12.4 \%$ with a range of $1.0 \%-41.9 \%$. SSIs following cesarean section in this area was $15.6 \%,{ }^{7}$ and $93 \%$ of the infection were superficial. ${ }^{8}$ The incidence of SSI was consistently higher $(23.2 \%)$ in

\footnotetext{
* Corresponding author.

E-mail address: bayekelkay@yahoo.com (B.K. Rade).
} 
low-human development index countries, even after adjusting for factors describing patients, diseases, procedures, safety, and hospitals. ${ }^{9}$ Over uses of antibiotics particularly in the postoperative period is associated with increased antimicrobial resistance and contribute for increment of SSI. ${ }^{10}$ The cost of SSI in terms of mortality, morbidity, health-care costs, and loss of productivity is enormous. ${ }^{8}$ Significant effect on quality of life for the patient, extended hospital stays, ${ }^{11}$ and cost burden health care system as personal and family level. ${ }^{12}$ Most SSIs are preventable. Different measures can be taken in the pre-operative phase (like; preoperative showering, avoid frequent vaginal examination, nasal decolonization, hair removal, patient theatre wear, staff theatre wear, staff leaving the operating area, hand jewellery, artificial nails and nail polish, antibiotic prophylaxis), intra-operative phase (hand decontamination, Sterile gowns, incise drapes, antiseptic skin preparation, maintaining patient homeostasis, antiseptics and antibiotics before wound closure, wound dressings, and closure methods) and post-operative phases of care (like; hanging dressings, postoperative cleansing, antibiotic treatment of surgical site infection and treatment failure) can reduce risk of infection. ${ }^{11}$

Studies in different parts of the world showed that preterm delivery, ${ }^{13}$ multiple vaginal examination, ${ }^{14,15}$ mothers from rural setting, ${ }^{16}$ prolonged labor, ${ }^{15-18}$ prolonged rupture of membrane, ${ }^{15,17}$ premature rupture of membrane (PROM), ${ }^{16}$ chorioamnionitis, ${ }^{16-18}$ smoking, ${ }^{13}$ hypertensive disorder, ${ }^{14,18}$ severe anemia, ${ }^{14,15,17}$ reactive in human immune deficiency virus, ${ }^{16}$ diabetes mellitus (DM), ${ }^{18}$ increase blood loss during surgery, ${ }^{16,19}$ and operation performed by junior physicians ${ }^{14,17}$ were the independent factors frequently associated with SSI following cesarean delivery.

Post cesarean wound sepsis reduction helpful to minimize the unnecessary morbidity and associated socioeconomic consequences for the patient and her family. ${ }^{20}$ Hence, finding risk factors to SSI and prevent accordingly will be vital to reduce occurrence of SSI and its consequences because majority of SSI are preventable. However, the studies regarding to this specific topic is limited in Gondar referral hospital which is cesarean section as the most common surgical procedure. Therefore, this study aimed to assess the magnitude SSI after cesarean delivery and associated factors among mothers who gave birth through cesarean section at University Gondar comprehensive and specialized hospital from January 1, 2018 to January 1, 2019.

\section{Methods and materials}

\subsection{Study design, area, and period}

A retrospective cross-sectional chart review was employed among mothers who underwent cesarean delivery in University of Gondar comprehensive and specialized hospital found in Gondar city. Gondar is one of a few metropolitan cities in Ethiopia, found about $741 \mathrm{Km}$ Northwest of Addis Ababa, the country's capital city. University of Gondar comprehensive and specialized hospital is one of the biggest tertiary level teaching hospitals. According to records from the hospital's information center, every year more than 200,000 people visit this hospital. In addition, this hospital used as research and practical teaching center for Medicine and other health science students with different specialty programs. Different gynecologic and obstetric procedures including elective cesarean section, emergency cesarean section and gynecologic surgeries are done in operation rooms found at urogynecology ward, main operation theater and labor ward. The data was collected from April 1, 2019 to May 30, 2019.

\subsection{Source and study populations}

All mothers who gave birth by cesarean section at University of Gondar comprehensive and specialized hospital were source population, and those who gave birth by cesarean section in this hospital from January 1st, 2018 to January 1st, 2019 were study population.

\subsection{Sample size determination and sampling procedure}

The sample size was estimated using a single population formula with the following assumptions: confidence level of $95 \%$; $2 \%$ margin of error; and $9.4 \%$ proportion of surgical site infection following cesarean delivery that was conducted at Felege-hiwot Specialized Hospital. ${ }^{18}$ With these assumptions, the calculated sample size for this study was 818. A total of 3210 mothers gave birth through cesarean section in this hospital for the last one year preceding to this study. Then, all mothers' chart identified and listed in ascending order by using their card numbers from operation registration book and check its completeness. Then, 2954 complete maternal charts were assured and systematic sampling technique was employed to select the candidate charts with sampling interval $(\mathrm{K})$ of three.

\subsection{Data collection and analysis}

Data were abstracts from mothers who gave birth by cesarean section in University of Gondar comprehensive and specialized hospital from January 1st, 2018 to January 1st, 2019. A total of 818 maternal charts were abstracted to access necessary data using well-structured abstraction checklist which was adapted from similar studies conducted in various parts of the world. The checklist consisted of three main items: socio-demographic related characteristics of mothers, maternal (obstetrical medical) related factors, and surgery (anesthesia and operation) related factors were extracted from maternal charts. Six (6) trained general practitioner physicians were recruited for data extraction, and two, year two resident (R-II) physician students assigned as supervisors. Completeness of data were checked on daily basis.

The extracted data were cleaned, coded, and entered Epi-info version 7.0, then exported to SPSS version 20 for analysis. Descriptive statistics were employed, and the results were presented in text and tables. Then, bivariate and multivariate logistic regression were applied orderly to find significant variables associated with SSI with P-value $<0.25$ and 0.05 , respectively. We also checked multicollinearity between variables by using a variance inflection factor (VIF) with the value of greater than 10 were not taken into the final model. The goodness-of-fit was checked by using the Hosmer-Lemeshow test. Finally, Variables with p-value $<0.05$ were considered as statistically significant.

\subsubsection{Operational definition}

Post cesarean section Surgical Site Infection: An infection which is developed after cesarean delivery on the operational site which is diagnosed by clinician. ${ }^{3}$

Prolonged hospital stays: hospital stay lasting more than 7 days.

Prolonged operation time: defined as cesarean section lasting more than $1 \mathrm{~h}$ from skin incision to last skin stitch.

\section{Results}

\subsection{Socio-demographic characteristics}

A total of 818 women's chart were enrolled in this study. The mean age of the women was 27.2 years with SD of \pm 5.9 years, and 590 $(72.2 \%)$ were with the age range of 20-34 years followed by 136 $(16.6 \%) \geq 35$ years of age. Four-hundred sixty-four $(56.7 \%)$ of the participants were living in an urban area, and 806 (98.5\%) of them were married.

\subsection{Maternal (obstetric and medical) related factors}

Two-fifth (39.9\%) of the women were Primi-para and 726 (88.8\%) of the mothers had ANC follow up history. About 396 (48.4\%) of them had rupture of membrane prior to the surgery. Of these, $92(23.3 \%)$ of them had rupture of membrane more than $12 \mathrm{~h}$ and 100 (14.6\%) of women were having more than $24 \mathrm{~h}$ of duration of labor. Around two-third of 
women having term pregnancy and one-fourth of participants (27.6\%) had meconium-stained amniotic fluid. Related to medical diseases, eighty-seven (10.6\%) mothers developed pregnancy-induced hypertension, 65 (7.9\%) mothers had anemia and 19 (2.3\%) women were HIV positive (Table 1).

\subsection{Anesthesia and operation-related characteristics}

All women underwent cesarean delivery were given prophylaxis antibiotics within $30 \mathrm{~min}$ before operation. About 688 (84.1\%) cesarean sections were emergency and $742(90.7 \%)$ of skin closure technique was subcuticular. All the cesarean sections were finished within $1 \mathrm{~h}$, almost all $98.8 \%$ and $99 \%$ were having estimated blood loss less than or equal to $1 \mathrm{~L}$ and taken spinal anesthesia respectively (Table 2).

\subsection{Magnitude of SSI and indications for cesarean section}

The overall magnitude of surgical site infection in this study was 100 (12.2\%). Regarding the type of surgical site infection; about 88 (88\%) of infections were superficial and $12(12 \%)$ were deep. Of 100 mothers who developed SSI following cesarean delivery, $60 \%$ were detected after discharge when mothers came back for follow up or complain. All of SSIs were detected within two weeks of post-operative days.

Table 1

Obstetrical and medical related characteristics of women underwent cesarean section at University of Gondar comprehensive and specialized hospital, Amhara Regional State, Ethiopia, 2019 ( $\mathrm{n}=818)$.

\begin{tabular}{|c|c|c|c|}
\hline Variables & Category & Frequency & $\begin{array}{l}\text { Percentage } \\
(\%)\end{array}$ \\
\hline \multirow[t]{3}{*}{ Parity } & $\begin{array}{l}\text { Primipara } \\
\text { (para1) }\end{array}$ & 326 & 39.9 \\
\hline & Para 2-4 & 366 & 44.7 \\
\hline & $\begin{array}{l}\text { Grand multipara } \\
(\geq 5)\end{array}$ & 126 & 15.4 \\
\hline \multirow[t]{2}{*}{ ANC Follow up } & Yes & 726 & 88.8 \\
\hline & No & 92 & 11.2 \\
\hline \multirow{3}{*}{$\begin{array}{l}\text { If "yes" Number of visits(n = } \\
726)\end{array}$} & 1 time & 10 & 1.4 \\
\hline & 2-4 times & 494 & 67.7 \\
\hline & $\geq 5$ times & 226 & 31 \\
\hline \multirow[t]{3}{*}{ Onset of labor } & Induced & 128 & 15.6 \\
\hline & Spontaneous & 558 & 68.2 \\
\hline & No labor & 132 & 16.1 \\
\hline \multirow{2}{*}{$\begin{array}{l}\text { Duration of labor in hours }(\mathrm{n}= \\
686 \text { ) }\end{array}$} & $<24$ & 586 & 85.4 \\
\hline & $\geq 24$ & 100 & 14.6 \\
\hline \multirow{2}{*}{$\begin{array}{l}\text { Duration of ROM before C/S in } \\
\text { hours }(n=396)\end{array}$} & $<12$ & 304 & 76.8 \\
\hline & $\geq 12$ & 92 & 23.2 \\
\hline \multirow[t]{4}{*}{ Gestational age } & Preterm & 72 & 8.8 \\
\hline & Term & 524 & 64.1 \\
\hline & Post-term & 82 & 10 \\
\hline & Unknown & 140 & 17.1 \\
\hline \multirow[t]{2}{*}{ Presence of meconium } & Yes & 226 & 27.6 \\
\hline & No & 592 & 72.4 \\
\hline \multirow[t]{2}{*}{ Presence of chorioamnionitis } & Yes & 15 & 1.83 \\
\hline & No & 803 & 98.17 \\
\hline \multirow{2}{*}{$\begin{array}{l}\text { Pregnancy-induced } \\
\text { hypertension }\end{array}$} & Yes & 87 & 10.6 \\
\hline & No & 731 & 89.4 \\
\hline \multirow[t]{2}{*}{ Anemia } & Yes & 65 & 7.9 \\
\hline & No & 753 & 92.1 \\
\hline \multirow[t]{2}{*}{ Diabetes mellitus } & Yes & 28 & 3.4 \\
\hline & No & 790 & 96.6 \\
\hline \multirow[t]{2}{*}{ HIV/AIDS } & Yes & 19 & 2.3 \\
\hline & No & 799 & 97.7 \\
\hline \multirow[t]{2}{*}{ On HAART } & Yes & 17 & 89.4 \\
\hline & No & 2 & 10.6 \\
\hline \multirow[t]{2}{*}{ Pneumonia } & Yes & 34 & 4.2 \\
\hline & No & 784 & 95.8 \\
\hline \multirow[t]{2}{*}{ UTI } & Yes & 26 & 3.2 \\
\hline & No & 792 & 96.8 \\
\hline
\end{tabular}

Abbreviations: ANC; Antenatal Care, ROM; Rupture of Membrane, C/S; Cesarean Section, HIV/AIDS; Human immunodeficiency virus, HAART; Highly Active Antiretroviral Therapy, UTI; Urinary Tract Infection.
Table 2

Anesthesia and operation-related characteristics of women underwent cesarean section at University of Gondar comprehensive and specialized hospital, Amhara Regional State, Ethiopia, $2019(\mathrm{n}=818)$.

\begin{tabular}{llll}
\hline Variable & Category & Frequency & Percentage (\%) \\
\hline Circumstance of surgery & Emergency & 688 & 84.1 \\
& Elective & 130 & 15.9 \\
Type of anesthesia & Spinal & 810 & 99 \\
& General & 8 & 1 \\
Type of skin incision & Pfannenstiel & 726 & 88.8 \\
& Midline & 92 & 11.2 \\
Type of skin closure & Interrupted & 76 & 9.3 \\
& Subcuticular & 742 & 90.7 \\
Thickness of subcutaneous & $\leq 2$ cm & 778 & 95.1 \\
tissue & $>2$ cm & 40 & 4.9 \\
Level of surgeon & Year 4 resident & 230 & 28.1 \\
& Year 3 resident & 273 & 33.3 \\
Post-operation hematocrit & Year 2 resident & 316 & 38.6 \\
& $\geq 30 \%$ & 753 & 92.1 \\
Intra-operative blood loss & $<30 \%$ & 65 & 7.9 \\
& $\leq 1000 \mathrm{ml}$ & 816 & 99.8 \\
\hline
\end{tabular}

Among mothers who underwent cesarean section, non-reassuring fetal heart rate pattern $194(23.7 \%)$ was the most common indication followed by previous cesarean section scar 138(16.9\%) and latent first stage of labor or early active phase of first stage of labor grade 2 or 3 meconium-stained amniotic fluid 122 (14.9\%) (Fig. 1).

\subsection{Factors associated with surgical site infection}

There were 9 independent variables in binary logistic regression which had $\mathrm{p}<0.25$ and became candidate for multivariable regression: duration of labor, rupture of membrane before surgery, chorioamnionitis, diabetes mellitus, HIV/AIDS, pregnancy-induced hypertension, anemia, meconium-stained amniotic fluid, and circumstance of surgery. After controlling all confounders in the multivariable analysis maternal (obstetric and medical) factors like: chorioamnionitis, diabetes mellitus, anemia, meconium-stained amniotic fluid, and duration of rupture of membrane more than $12 \mathrm{~h}$ before cesarean section were significantly associated with $p<0.05$.

Mothers who had chorioamnionitis were 6.46 times more likely to develop surgical site infection as compared to their counterparts [AOR $=6.46,95 \% \mathrm{CI}=1.82-22.71]$. Those mothers who had diabetes mellitus were 6.02 more likely to develop surgical site infection than nondiabetic mother $[\mathrm{AOR}=6.02,95 \% \mathrm{CI}=1.69-21.36]$. Mothers who were having more than $12 \mathrm{~h}$ of rupture of membrane before cesarean section were 2.94 times more likely at risk for surgical site infections than less than $12 \mathrm{~h}$ of rupture of membrane $[\mathrm{AOR}=2.94,95 \% \mathrm{CI}=$ 1.52-5.67]. Mothers who had meconium-stained amniotic fluid (MSAF) during labour delivery and being anemic were 2.43 and 3.44 times more likely to develop SSI following CS compared to their counterparts with $[\mathrm{AOR}=2.43,95 \% \mathrm{CI} 1.23-4.81]$ and $[\mathrm{AOR}=3.44,95 \% \mathrm{CI}=$ 1.56-7.56] respectively (Table 3 ).

\section{Discussion}

The magnitude of surgical site infection following cesarean section in this study was $12.2 \%$ with [95\% CI (9.6-14.44)]. The magnitude of SSI in this study was in line with the study finding at Tanzania $(10.9 \%)^{14}$ and various parts of Ethiopia: Hawassa (11\%), Mekelle (11.7\%), and Jimma (1.4\%). ${ }^{16,17,21}$ The similarities could be due to the study setting, patients' background and surgeons who performed the operations. However, the finding was higher than the value obtained from United States $5.5 \%,{ }^{13}$ and Oman $(2.66 \%){ }^{22}$ It was also higher than the endogenous findings of Bahirdar (9.4\%), ${ }^{18}$ Miachew $(6.8 \%),{ }^{3}$ and Assela $(9.4 \%){ }^{23}$ The reason may be due to the difference of socio-economic status, quality of care and level awareness between developing and 


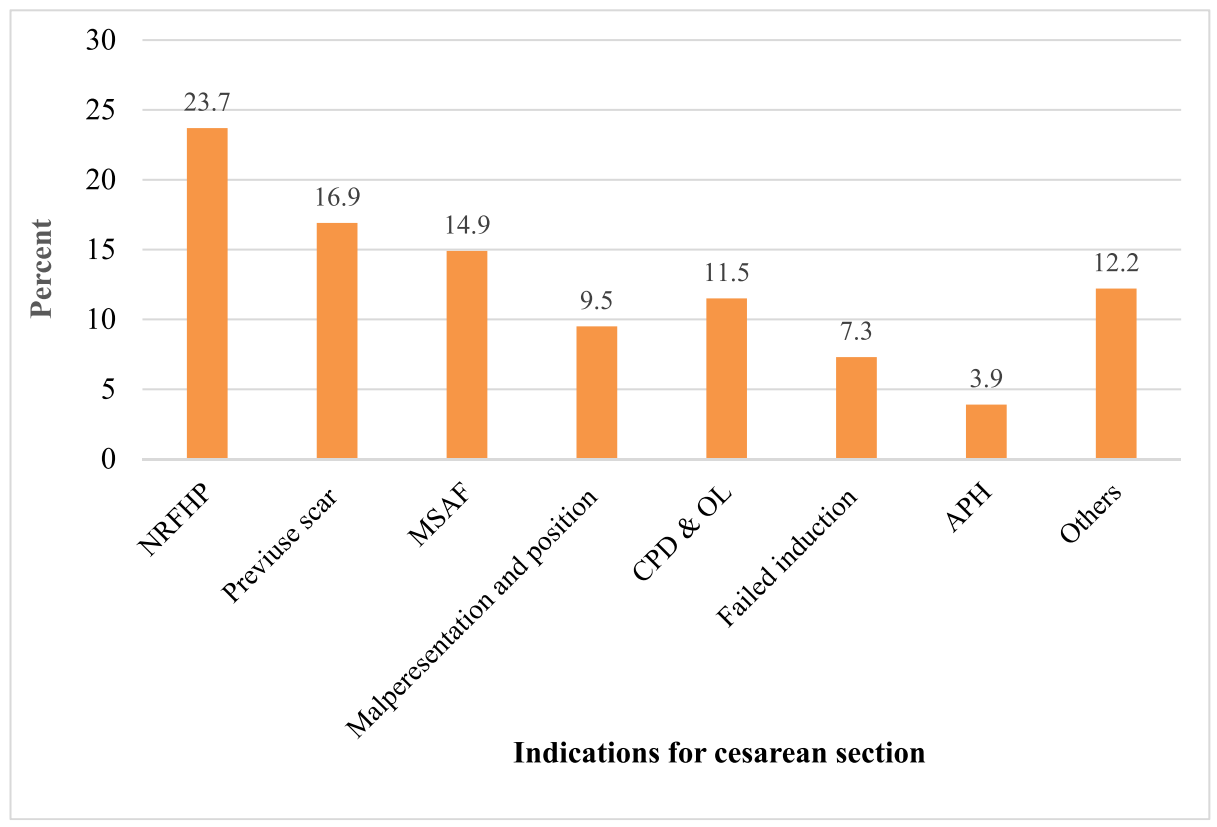

Fig. 1. This graph shows the distribution of indications for cesarean section done at University of Gondar comprehensive and specialized hospital, Amhara Regional State, Ethiopia, 2019.

Abbreviations: NRFHR; Non reassuring fetal heart rate pattern, MSAF; Meconium-stained amniotic fluid, CPD \& OL; Cephalo-pelvic disproportion and obstructed labour, APH; antepartum hemorrhage and others; Sever oligohydramnios, cervical arrest, cord prolapse, fetal macrosomia and previous fistula repair.

Abbreviations: NRFHR; Non reassuring fetal heart rate pattern, MSAF; Meconium-stained amniotic fluid, CPD \& OL; Cephalo-pelvic disproportion and obstructed labour, APH; antepartum hemorrhage and others; Sever oligohydramnios, cervical arrest, cord prolapse, fetal macrosomia and previous fistula repair.

Table 3

The bivariate and multivariable association of surgical site infections and independent factors among women who underwent cesarean section at University of Gondar comprehensive and specialized hospital, Amhara Regional State, Ethiopia, 2019.

\begin{tabular}{|c|c|c|c|c|c|}
\hline \multirow[t]{2}{*}{ Characteristics } & \multicolumn{2}{|c|}{ Surgical sit infection } & \multirow[t]{2}{*}{ COR $(95 \% \mathrm{CI})$} & \multirow[t]{2}{*}{ AOR $(95 \% \mathrm{CI})$} & \multirow[t]{2}{*}{ P-value } \\
\hline & No & Yes & & & \\
\hline \multicolumn{6}{|c|}{ Meconium-stained amniotic fluid } \\
\hline No & $545(92 \%)$ & $47(8 \%)$ & 1.00 & 1.00 & \\
\hline Yes & $173(76.5 \%)$ & $53(23.5 \%)$ & $3.55(2.31-5.45)$ & $2.43(1.23-4.81)$ & $0.011^{* *}$ \\
\hline \multicolumn{6}{|l|}{ Chorioamnionitis } \\
\hline No & $712(88.7 \%)$ & $91(11.3 \%)$ & 1.00 & 1.00 & \\
\hline Yes & $6(37.5 \%)$ & $9(62.5 \%)$ & $11.7(4.68-37.14)$ & $6.46(1.82-22.7)$ & $0.01 * *$ \\
\hline \multicolumn{6}{|c|}{ Circumstance of surgery } \\
\hline Emergency & $592(86 \%)$ & $96(14 \%)$ & $5.10(1.85-14.15)$ & $1.08(0.89-1.19)$ & 0.999 \\
\hline Elective & $126(96.9 \%)$ & $4(3.1 \%)$ & 1.00 & 1.00 & \\
\hline \multicolumn{6}{|c|}{ Post -operative hematocrit } \\
\hline$\geq 33 \%$ & $677(89.9 \%)$ & $76(10.1 \%)$ & 1.00 & 1.00 & \\
\hline$<33 \%$ & $41(63 \%)$ & $24(37 \%)$ & $5.21(2.99-9.00)$ & $3.44(1.56-756)$ & $0.002 * *$ \\
\hline \multicolumn{6}{|l|}{ Diabetes mellitus } \\
\hline No & $700(88.6 \%)$ & $90(11.4 \%)$ & 1.00 & 1.00 & \\
\hline Yes & $18(64.2 \%)$ & $10(35.8 \%)$ & $4.32(1.93-9.65)$ & $6.02(1.69-21.36)$ & $0.005^{* *}$ \\
\hline \multicolumn{6}{|l|}{ HIV/AIDS } \\
\hline No & $704(88.1 \%)$ & $95(11.9 \%)$ & 1.00 & 1.00 & \\
\hline Yes & $14(73.6 \%)$ & $5(26.4 \%)$ & $2.64(0.93-7.51)$ & $2.96(0.84-10.4)$ & 0.09 \\
\hline \multicolumn{6}{|c|}{ Pregnancy induced hypertension } \\
\hline No & $647(88.5 \%)$ & $84(11.5 \%)$ & 1.00 & 1.00 & \\
\hline Yes & $71(81.6 \%)$ & $16(18.4 \%)$ & $1.76(0.96-3.12)$ & $2.11(0.86-5.17)$ & 0.101 \\
\hline \multicolumn{6}{|c|}{ Duration of labor in hours } \\
\hline$\leq 24$ & $522(88.3 \%)$ & $66(11.7)$ & & & \\
\hline$>24$ & $70(70 \%)$ & $30(30 \%)$ & $3.39(2.05-5.55)$ & $1.26(0.62-2.58)$ & 0.52 \\
\hline \multicolumn{6}{|c|}{ Duration of rupture of membrane before cesarean section in hours } \\
\hline$\leq 12$ & $266(87.5 \%)$ & $38(12.5 \%)$ & 1.00 & 1.00 & \\
\hline$>12$ & $60(66.7 \%)$ & $30(33.3 \%)$ & $3.73(2.16-6.45)$ & $2.94(1.52-5.67)$ & $0.001^{* *}$ \\
\hline
\end{tabular}

Note: ** Significantly associated variables with $\mathrm{P}$ value $<0.05,1.00$ : reference variable. 
developed countries. Whereas, the difference with endogenous findings could be due to flow of patients. In fact, university of Gondar hospital delivery report showed that there were around 1000 deliveries per month which was not comparable with the number of beds the hospital had. So that, patient overcrowding might have an impact for spreading of causative agents for infections. On the other side, this finding was lower than the value obtained from Uganda $(16.4 \%),{ }^{24}$ and Malaysia $(18.8 \%) .{ }^{19}$ This discrepancy could be due to the study design. We have used charts to access the data but the above-mentioned studies used observational follow-up study design. Therefore, observational follow-up study helps to get more patients who developed SSIs after discharge and those who visited other health facilities for the infections.

There were five independent maternal related factors (three obstetrical and two medical) were significantly associated with SSI. Duration of rupture of membrane more than $12 \mathrm{~h}$ before cesarean section was an independent risk factor for surgical site infection. This finding is supported with study conducted in Oman. ${ }^{22}$ It is also similar with the study done in Ethiopia Miachew, Mekelle, Hawassa, and Assela. ${ }^{3,16,17,23}$ The possible explanation could be, membrane is one of protective barrier for ascending infection. When membrane rupture, this protection is lost and poly-microbial organisms easily ascend during vaginal examination and cause infection during uterine and skin incision. Diabetes mellitus was noted to be another independent risk factor for surgical site infection. It was supported by other studies done in Oman and India. ${ }^{22,25}$ Also, similar with the findings of Ethiopia, Bahirdar and Assela. ${ }^{18,23}$ This may be due to increasing glucose level in the blood exerts multiple opposing effects on well recognized cellular immunity. Most importantly, acute, short-term hyperglycemia affects all major components of innate immunity and impairs the ability of the host to combat infection. In case of long-standing diabetes mellites; vascular complications that impairs adequate tissue perfusion, and oxygenation is crucial factor for wound healing process.

In this study chorioamnionitis was also another independent factor associated with surgical site infection. This finding is consistent with the study conducted in Ethiopia; Mekelle, Bahirdar, Jimma, and Assela. ${ }^{16,18,21,23}$ This might be explained by the fact that once the amniotic fluid is contaminated, during cesarean section this fluid will have leakage and contamination with peritoneum and skin incision which predisposed the incision site to be infected. The presence of meconium-stained amniotic fluid was strongly associated with increased risk of surgical site infection. This finding was consistent with the study done in Jimma. ${ }^{21}$ The plausible explanation is that contaminated incision site with thick meconium serves as good media for bacterial proliferation.

Finally, post-operative hematocrit less than 33\% (anemia) was significantly associated with surgical site infection. This finding was agreed with the study done in Oman, ${ }^{22}$ Tanzania, ${ }^{14}$ Hawassa, ${ }^{17}$ and Assela. ${ }^{23}$ In general, low hematocrit concentration reduces the oxygen tension in the wound and increases the risk of wound infection by compromising the activity of macrophages and impeding wound healing progress.

\subsection{Limitation}

This study has all drawbacks of cross-sectional study design and using secondary data. In addition, patients were not followed after being discharged from the hospital.

\subsection{Prior evidences and added value of this study}

The problem's magnitude and the risk factors from the previous studies were heterogenous. Infrastructure (facility) and sociodemographic related factors were frequently influenced the occurrence of surgical site infection following cesarean delivery. Involving multiple sectors effort and working for long time are needed to prevent the aforementioned factors. However, in this study, only maternal (obstetrical and medical) factors were identified as influencing factors that are easily preventable. The obstetrical factors (prolonged PROM, chorioamnionitis, and meconium-stained amniotic fluid) can be prevented by proper risk assessment and management during ANC follow up and within care providers scope of management skill. Additionally, this study proves the prior studies and helps to reach scientific consensuses, which factors come to the clinicians' mind when they provide obstetrical care to prevent infections. Again, this finding is new (up-todated) compared with the previous national and international reports.

\section{Conclusion}

The magnitude of surgical site infection following cesarean section was high compared to the standard CDC guidelines of surgical site infection. Maternal related factors including obstetrical (prolonged rupture of membrane prior to cesarean section, chorioamnionitis and meconium-stained amniotic fluid), and medical factors (diabetes mellitus and anemia) were found significantly associated with surgical site infections following cesarean section. Improvement of early identification of risk factors, and prompt treatment/control of obstetrical and medical complications are the recommendations. To understand more about the nature of the problem, further observational studies set as recommendation as well.

\section{Ethical approval and consent to participate}

Ethical clearance was obtained from institutional ethical review board of University of Gondar (Ref No: SOM/1368/2019). Consent for the record of review was obtained from institute review board and University of Gondar comprehensive specialized hospital. Records were coded and kept confidential. The data were not be used for any purpose other than the study aims. Lastly, we confirm that this study was conducted in accordance with the Declaration of Helsinki.

\section{Availability of the data and materials}

The datasets used and/or analyzed during the current study are available from corresponding author upon on reasonable request.

\section{Funding}

This research did not receive any specific grant from funding agencies, commercial or not-for-profit sectors.

\section{Declaration of competing interest}

The authors declare that they have no competing interests.

\section{Acknowledgment}

We would like to acknowledge University of Gondar comprehensive and specialized hospital, data extractors and supervisors.

$\begin{array}{ll}\text { Abbreviations } \\ \text { ANC } & \text { Antenatal care } \\ \text { AOR } & \text { Adjusted odds ratio } \\ \text { CI } & \text { Confidence interval } \\ \text { COR } & \text { Crude odds ratio } \\ \text { CS } & \text { cesarean section } \\ \text { PROM } & \text { premature rupture of membrane } \\ \text { ROM } & \text { Rupture of membrane } \\ \text { SSI } & \text { Surgical site infection }\end{array}$




\section{References}

1 Horan TC, Gaynes RP, Martone WJ, Jarvis WR, Emori TG. CDC definitions of nosocomial surgical site infections, 1992: a modification of CDC definitions of surgical wound infections. Infect Control Hosp Epidemiol. 1992;13(10):606-608.

2 Berríos-Torres SI, Umscheid CA, Bratzler DW, et al. Centers for disease control and prevention guideline for the prevention of surgical site infection. JAMA Surg. 2017; 152(8):784-791. https://doi.org/10.1001/jamasurg.2017.0904, 2017.

3 Gelaw KA, Aweke AM, Astawesegn FH, Demissie BW, Zeleke LB. Surgical site infection and its associated factors following cesarean section: a cross-sectional study from a public hospital in Ethiopia. Patient Saf Surg. 2017;11:18.

4 World Health Organization. WHO statement on caesarean section rates. http://apps. who.int/iris/bitstream/handle/10665/161442/WHO_RHR_15.02_eng.pdf;jsessioni $\mathrm{d}=$ E8BFDF44D047A17ADDC1387AD480C0B1? sequence $=1 ; 2015$

5 Boerma T, Ronsmans C, Melesse DY, et al. Global epidemiology of use of and disparities in cesarean sections. Lancet. 2018;392(10155):1341-1348.

6 The Lancet. Stemming the global caesarean section epidemic. Lancet. 2018 Oct 13; 392(10155):1279. https://doi.org/10.1016/S0140-6736(18)32394-8. PMID: 30322560 .

7 Angie Sway KK, Anthony Wanyoro, Claire Kilpatrick, Joseph Solomkin. Review of the Burden of Infection Following Cesarean Section in Sub-saharan Africa. 23 April 2017.

8 Chu K, Maine R, Trelles M. Cesarean section surgical site infections in sub-Saharan Africa: a multi-country study from Medecins Sans Frontieres. World J Surg. 2015;39 (2):350-355.

9 Bradley van Paridon. Incidence of surgical site infections highest in lower-income countries. https://www.infectiousdiseaseadvisor.com/home/topics/nosocomial infections/incidence-of-surgical-site-infections-highest-in-lower-income-countries/; 2018.

10 Sawyer RG, Evans HL. Surgical site infection - the next frontier in global surgery. Lancet Infect Dis. 2018;18(5):477-478.

11 Surgical Site Infections: Prevention and Treatment. national institute for health and care excellence; 22 October 2008.

12 Saeed KBM, Greene RA, Corcoran P, Neill SM. Incidence of surgical site infection following cesarean section: a systematic review and meta-analysis protocol. BMJ Open. 2017;7(1), e013037.

13 Moulton LJ, Munoz JL, Lachiewicz M, Liu X, Goje O. Surgical site infection after cesarean delivery: incidence and risk factors at a US academic institution. J Matern Fetal Neonatal Med. 2018;31(14):1873-1880.

14 Mpogoro FJ, Mshana SE, Mirambo MM, Kidenya BR, Gumodoka B, Imirzalioglu C. Incidence and predictors of surgical site infections following cesarean sections at
Bugando Medical Centre, Mwanza, Tanzania. Antimicrob Resist Infect Control. 2014;3: 25.

15 Gelaw Misganaw Worku, Ahmed Abdella. Prevalence of surgical site infection and associated factors among mothers after cesarean delivery in zewditu memorial hospital. Ethiopian J Reprod Health (EJRH). 2018;10(4).

16 Wendmagegn TA, Abera GB, Tsehaye WT, Gebresslasie KB, Tella BG. Magnitude and determinants of surgical site infecion among women underwent cesarean section in Ayder comprehensive specialized hospital Mekelle City, Tigray region, Northern Ethiopia. BMC Pregnancy Childbirth. 2016;18(1):489, 2018.

17 Wodajo S, Belayneh M, Gebremedhin S. Magnitude and factors associated with postcesarean surgical site infection at Hawassa university teaching and referral hospital, Southern Ethiopia: a cross-sectional study. Ethiopian J Health Sci. 2017;27(3): 283-290.

18 Gedefaw G, Asires A, Shiferaw S, Addisu D. Factors associated with surgical site infection among women undergoing obstetrics surgery at Felegehiwot Referral Hospital, Bahir Dar, Northwest Ethiopia: a retrospective cross-sectional study. Saf Health. 2018;4(1):14.

19 Jasim HH, Sulaiman SAS, Khan AH, Dawood OT, Abdulameer AH, Usha R. Incidence and risk factors of surgical site infection among patients undergoing cesarean section. Clin Med Insights Ther. 2017;9, 1179559X17725273.

20 Gideon K, Kurigamba DN, Nanyanga Irene, Nahabwe Haven, Mutahunga Birungi R. Post-ceserean wound sepsis: recognizable risks and causes at a rural Ugandan hospital. Int J Women's Health Care. 25 July 2018;3(2):1-4.

21 Amenu D, Belachew T, Araya F. Surgical site infection rate and risk factors among obstetric cases of jimma university specialized hospital, southwest Ethiopia. Ethiopian J Health Sci. 2011;21(2):91-100.

22 Dhar H, Al-Busaidi I, Rathi B, Nimre EA, Sachdeva V, Hamdi I. A study of postcesarean section wound infections in a regional referral hospital, Oman. Sultan Qaboos Univ Med J. 2014;14(2):e211-e217.

23 Mamo T, Abebe TW, Chichiabellu TY, Anjulo AA. Risk factors for surgical site infections in obstetrics: a retrospective study in an Ethiopian referral hospital. Patient Saf Surg. 2017;11(1):24.

24 Lubega A, Bazira J, Lucy N. Incidence and etiology of surgical site infections among emergency postoperative patients in Mbarara regional referral hospital, South Western Uganda. Surg Res Pract. 2017:1-6, 2017.

25 Pathak A, Mahadik K, Swami MB, et al. Incidence and risk factors for surgical site infections in obstetric and gynecological surgeries from a teaching hospital in rural India. Antimicrob Resist Infect Control. 2017;6(1):66. 